\title{
White matter microstructure in schizophrenia: effects of disorder, duration and medication
}

Richard Kanaan, Gareth Barker, Michael Brammer, Vincent Giampietro, Sukhwinder Shergill, James Woolley, Marco Picchioni, Timothea Toulopoulou and Philip McGuire

\section{Background}

Diffusion tensor magnetic resonance imaging studies in schizophrenia to date have been largely inconsistent. This may reflect variation in methodology, and the use of small samples with differing illness duration and medication exposure.

\begin{abstract}
Aims
To determine the extent and location of white matter microstructural changes in schizophrenia, using optimised diffusion tensor imaging in a large patient sample, and to consider the effects of illness duration and medication exposure.
\end{abstract}

\section{Method}

Scans from 76 patients with schizophrenia and 76 matched controls were used to compare fractional anisotropy, a measure of white matter microstructural integrity, between the groups.

\section{Results}

We found widespread clusters of reduced fractional anisotropy in patients, affecting most major white matter tracts. These reductions did not correlate with illness duration, and there was no difference between age-matched chronically and briefly medicated patients.

\section{Conclusions}

The finding of widespread fractional anisotropy reductions in our larger sample of patients with schizophrenia may explain some of the inconsistent findings of previous, smaller studies.

\section{Declaration of interest}

None. Funding detailed in Acknowledgements.
There is a growing focus on disconnectivity as a model for schizophrenia, ${ }^{1,2}$ following the evidence of functional disconnectivity from functional neuroimaging. This has led to a renewed interest in white matter, and in white matter imaging in particular. The results of volumetric magnetic resonance imaging (MRI) studies of white matter in schizophrenia have been inconclusive, however. Whereas grey matter analyses have shown robust changes and a clear relationship with disease progression, ${ }^{3}$ the extent of white matter changes and their relation to illness duration or the effects of medication remain in doubt. ${ }^{4}$

Diffusion tensor imaging (DTI) offers a method of examining white matter microstructure in vivo. ${ }^{5}$ It has proved effective in detecting subtle white matter pathology and is a promising method of investigating anatomical connectivity in schizophrenia. ${ }^{6}$ There have been a number of DTI studies in schizophrenia in recent years, but the results so far have been inconsistent with respect to both the location and extent of white matter abnormalities. This may reflect both a lack of power to detect differences, which seem to be small and highly variable, ${ }^{7}$ and methodological differences. Region of interest studies have selected different foci of investigation but have often been small. Voxel-based approaches permit assessment of the entire white matter, but the studies in schizophrenia to date have used a variety of different acquisition and analysis protocols. ${ }^{6}$

The first goal of our study was to use DTI to assess the microstructure of white matter in schizophrenia, as far as possible overcoming the methodological difficulties that may have limited previous DTI studies of this disorder. We therefore studied a large sample of patients and a carefully matched control group using a sophisticated acquisition sequence, optimised for the study of white matter, ${ }^{8}$ and a voxel-based method of analysis specifically developed for DTI data. Our main hypothesis was that with greater statistical power, and by examining all implicated tracts, we would reconcile the apparently inconsistent findings in the literature by revealing widespread abnormalities in schizophrenic white matter. We further predicted that our greater statistical power would reveal small negative relationships between illness duration, medication and white matter microstructure.

\section{Method}

Seventy-six patients meeting DSM-IV criteria for schizophrenia ${ }^{9}$ were recruited from the wards and out-patient clinics of the South London and Maudsley Hospital National Health Service (NHS) Trust and by national referral. Diagnoses were established by an experienced psychiatrist, using semi-structured interviews and detailed case-note review. Patients who were assessed within the first few months of their illness had the 6-month duration criterion confirmed at subsequent follow-up. The median duration of illness (defined as time from first contact with services) for all patients at assessment was 4.5 years (range 3 months to 35 years) and their median age was 27 years (range 18-60). All but 11 patients were receiving antipsychotic medication at the time of scanning. Seventy-six healthy volunteers were then selected from a sample of over 200 controls, to match the patient group for age, gender, handedness and premorbid IQ using the National Adult Reading Test. ${ }^{10}$ Control group volunteers were excluded if they had a personal history of mental illness or a family history of psychotic illness, and both patients and controls were excluded if there was a lifetime history of head injury with loss of consciousness, neurological illness or current drug or alcohol dependence. To examine the effects of antipsychotic medication on DTI data, a subgroup of 15 patients who had been treated with medication for less than 1 month (median 3 days, range 0-25) were matched for age, gender, IQ and handedness with another subgroup of 30 patients who had been treated for more than 1 year (median 3 years, range 1-13), 
selected without reference to their DTI results. Our sample was powered by the subgroup analysis to give $80 \%$ power to detect changes of the order described in the most recent large study for which suitable data were available. ${ }^{11}$ The power to detect changes of that order in the principal analysis was correspondingly over $99 \%$.

The study was approved by the appropriate NHS research ethics committee, and all participants gave written informed consent before taking part.

\section{Diffusion tensor data acquisition}

Data were acquired using a $1.5 \mathrm{~T}$ GE Signa LX system (General Electric, Milwaukee, USA) with actively shielded magnetic field gradients (maximum amplitude $40 \mathrm{mT} / \mathrm{m}$ ). A standard quadrature birdcage head coil was used for both radiofrequency transmission and signal reception. Each volume was acquired using a multislice, peripherally gated echoplanar imaging sequence, optimised for precise measurement of the diffusion tensor in parenchyma from healthy volunteer data, ${ }^{8}$ over 60 contiguous $2.5 \mathrm{~mm}$ thick nearaxial slice locations. Data were acquired with a $96 \times 96$ matrix over a $24 \mathrm{~cm} \times 24 \mathrm{~mm}$ field of view, yielding isotropic $(2.5 \mathrm{~mm} \times$ $2.5 \mathrm{~mm} \times 2.5 \mathrm{~mm}$ ) voxels, although during reconstruction the data were zero-filled to $128 \times 128$, giving an apparent in-plane voxel size of $1.875 \mathrm{~mm} \times 1.875 \mathrm{~mm}$. The echo time was $107 \mathrm{~ms}$ and the repetition time was $15 \mathrm{R}-\mathrm{R}$ intervals. The duration of the diffusion encoding gradients $(\delta)$ was $17.3 \mathrm{~ms}$, giving a maximum diffusion weighting of $1300 \mathrm{~s} / \mathrm{mm}^{2}$. At each slice location, seven images were acquired with no diffusion gradient applied, together with diffusion-weighted images in 64 gradient directions uniformly distributed in space (see Jones et al for further details). ${ }^{8}$

\section{Data processing}

The diffusion-weighted images were first corrected for eddy current distortion using a mutual information-based registration scheme, and then masked using locally written software plus the Brain Extraction Tool (BET) in the Functional Software Library package (Oxford Centre for Functional Magnetic Resonance Imaging of the Brain, Oxford University, UK). The diffusion tensor was then calculated at each voxel using multivariate linear regression after logarithmic transformation of the signal intensities. ${ }^{5}$ Fractional anisotropy (an index of white matter microstructural organisation) was calculated at each voxel to produce a multislice fractional anisotropy image. Normalisation (i.e. transformation of the scans into a standard space to allow inter-individual comparison) used a two-stage process. In the first step, a study-specific template was created and the fractional anisotropy images were then registered to this as follows: the mean $\mathrm{b}=0$ (non-diffusion-weighted) image from every participant was registered using SPM2 (Wellcome Department of Imaging Neuroscience, London, UK) to the SPM2 echoplanar imaging template. The derived mapping parameters for each participant were then applied to that person's (inherently co-registered) fractional anisotropy image. These normalised images were themselves averaged and smoothed with an $8 \mathrm{~mm}$ Gaussian kernel to create a study-specific template. The second stage involved a new registration, as the original fractional anisotropy images were then registered to this template, again using SPM2. The registered images were also segmented, using the default tissue probability information ('priors') in SPM2, and these probabilistic maps thresholded at $10 \%$ probability to generate a liberal white/restof-brain mask. The fractional anisotropy images were smoothed with a $5 \mathrm{~mm}$ (full width half maximum) kernel, before applying the white matter mask to create white-matter-only fractional anisotropy maps. Note that the smoothing was not to comply with the statistical requirements of parametric analysis, since the analysis stage uses non-parametric methods, but simply to increase signal:noise ratio - although this also served to sensitise the analysis to structures with spatial extents of this size. ${ }^{12}$ All computation was carried out on a Sun workstation (Sun Microsystems, Mountain View, California, USA).

\section{Statistical analysis}

The principal analysis was a voxel-based analysis of variance (ANOVA) of the fractional anisotropy of the white matter of the patient group compared with the control group. This was carried out in XBAM version 3.4 (Institute of Psychiatry, London, UK) employing a permutation-based method. The one-way ANOVA was fitted to each voxel of the normalised, segmented fractional anisotropy maps using patient $v$. control status as the grouping variable. The ANOVA was only fitted at voxels where all participants contributed; when combined with the liberal thresholding described earlier, this confined analysis to the body of the white matter. After fitting the ANOVA model to the observed data, the participant labels were randomly permuted between the two groups to achieve the null hypothesis of no main effect of group membership on fractional anisotropy. This permutation was carried out 1000 times at each voxel to allow the construction of a voxel-level null distribution of fractional anisotropy differences. This approach is necessary with DTI because in areas close to tissue boundaries any normalisation error will produce a strongly bimodal distribution of fractional anisotropy. A final advantage of the non-parametric approach is that hypotheses can be tested at the cluster level rather than at individual voxel level, potentially increasing sensitivity, and this level can be chosen so as to give precise control over the false positive rate. ${ }^{13}$ After determination of voxels showing significant effects at a relatively low threshold $(P<0.01)$, sets of spatially contiguous suprathreshold voxels were identified, and the sum of the suprathreshold voxel-wise test statistics (or 'mass') of each three-dimensional cluster was calculated. The mass of each cluster was then tested against the corresponding permutation distribution, an approach for which there is no parametric equivalent owing to the lack of appropriate theoretical distributions. ${ }^{14}$ Voxel and cluster-wise probability thresholds were chosen to ensure less than one false positive in the imaging volume. The identification of clusters with white matter tracts was made by reference to Mori et al and Crosby et al. ${ }^{15,16}$

The secondary analyses used the significant clusters identified by the principal analysis. The mean fractional anisotropy over each identified cluster was extracted for each participant. This allowed correlations of illness duration with fractional anisotropy, and comparison of the cluster means for the medicated $v$. unmedicated groups. These analyses were carried out using SPSS version 13.0 for Windows.

\section{Results}

The demographic characteristics of the sample are shown in Table 1 and compared with the patient group according to medication status in Table 2 .

The principal analysis revealed several white matter areas where the patient group had significantly lower fractional anisotropy than the control group (at thresholds of voxel $P<0.01$ and cluster $P<0.0025$, chosen so that less than one false positive cluster would be expected within the image volume by chance alone; Fig. 1). In contrast, there was no area where fractional anisotropy was higher in the patient group. Table 3 lists 


\begin{tabular}{|lccc|}
\hline Table 1 Demographic characteristics of the sample & & & $P^{\mathrm{a}}$ \\
\hline Age, years: mean (s.d.) & Patients $(n=76)$ & Controls $(n=76)$ & 0.9 \\
\hline Male/female, $n$ & $30.9(10.2)$ & $30.5(10.0)$ & 0.8 \\
\hline Handedness & $66 / 10$ & $65 / 11$ & 0.7 \\
\hline IQ, mean (s.d.) & All right-handed & All right-handed & \\
\hline IIIness duration, years: median & $105.2(9.7)$ & & \\
\hline a. All values represent Mann-Whitney tests. & 4 & & \\
\hline
\end{tabular}

\begin{tabular}{|lccc|}
\hline Table 2 & Demographic comparison of medicated $v$. only briefly medicated patients & & $P^{\mathrm{a}}$ \\
\hline Age, years: mean (s.d.) & Medicated $(n=30)$ & Unmedicated $(n=15)$ & 0.3 \\
\hline Male/female, $n$ & $25.8(5.3)$ & $24.1(5.2)$ & 0.4 \\
\hline Handedness & $26 / 4$ & $14 / 1$ & \\
\hline QQ, mean (s.d.) & All right-handed & All right-handed & 0.1 \\
\hline Treatment duration, median & $102.5(11.2)$ & $97.8(6.8)$ & $<0.0001$ \\
\hline a. All values represent Mann-Whitney tests. & 3 years & 3 days & \\
\hline
\end{tabular}

the coordinates of the centre of mass and approximate white matter location for each cluster. All but one of the clusters were extensive, however, and in no case corresponded to a single white matter structure. They are more fully described as follows.

\section{Cluster location}

Brainstem

A cluster of reduced fractional anisotropy lay within the brainstem, an area where several small white matter structures run close to each other. At its most inferior extent the cluster included the inferior cerebellar peduncle on the right, and extended to include the right superior cerebellar peduncle, and the lateral aspect of the cerebral peduncle at its most superior extent.

\section{Frontal}

A large area of reduced fractional anisotropy in the right inferior frontal white matter extended ventrally to include areas corresponding to parts of the inferior fronto-occipital fasciculus, the forceps minor and the anterior radiations of the corona radiata. More posteriorly the cluster extended bilaterally to include parts of the genu of the corpus callosum and the superior fronto-occipital fasciculus, and abutted the cingulum bundle, notably on the left.

\section{Medial}

The large right anterior cluster described above traced the lateral border of the corpus callosum as it forms the body. A second cluster lay in the anterior limb of the internal capsule on the right. A third lay where the interior and external capsules meet posteriorly on the left; this area may contain fibres from the left inferior longitudinal fasciculus, optic radiation and fornix.

\section{Lateral}

There were clusters in medial areas of the superior longitudinal fasciculus bilaterally. These were more extensive in the left hemisphere but extended more antero-superiorly in the right.

\section{Medial temporal/occipital}

Large bilateral clusters traced areas of white matter extending from the medial temporal lobes to the occipital pole. In form these most clearly corresponded to the inferior longitudinal fasciculi; however, the inferior fronto-occipital fasciculi, the optic radiations and the splenium of the corpus callosum - although they have origins superiorly and medially - could not be excluded from the occipital parts of the clusters.

\section{Superior corona radiata}

Bilateral areas of fractional anisotropy reduction were found in the superior radiations of the corona radiata.

\section{Whole brain white matter fractional anisotropy}

The mean fractional anisotropy over the whole brain white matter was significantly different between groups $(0.314$ in patients

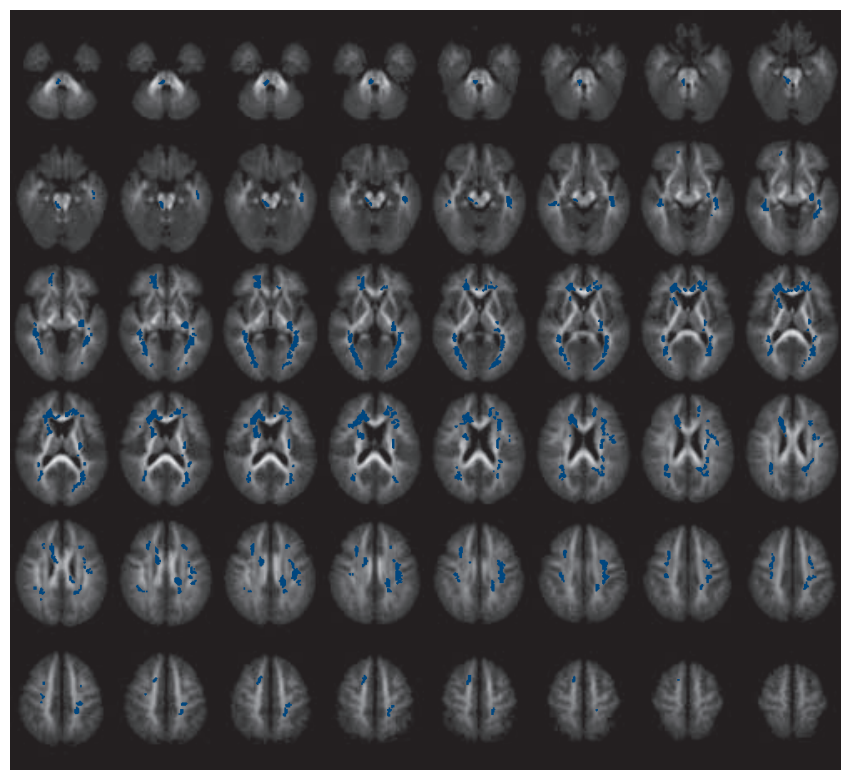

Fig. 1 Areas of reduced fractional anisotropy in patients with schizophrenia $v$. controls (the left of the brain is on the right of the slice images). 
Table 3 Areas of reduced fractional anisotropy in patients compared with controls

\begin{tabular}{|c|c|c|c|c|c|c|c|}
\hline \multirow[b]{2}{*}{ White-matter region } & \multirow[b]{2}{*}{ Side } & \multicolumn{3}{|c|}{ Talairach coordinates } & \multicolumn{2}{|c|}{ Fractional anisotropy, mean (s.d.) } & \multirow[b]{2}{*}{$P$} \\
\hline & & $x$ & $y$ & $z$ & Patient group & Control group & \\
\hline Brainstem & Right & 5 & -31 & -22 & $452(30)$ & $470(20)$ & 0.002 \\
\hline ILF & Right & 40 & -31 & -8 & 453 (29) & $472(26)$ & $<0.001$ \\
\hline Fornix & Left & -27 & -22 & -6 & $446(26)$ & $464(18)$ & 0.001 \\
\hline ILF/IFO/optic radiations & Right & 22 & -80 & -2 & $401(31)$ & $426(26)$ & $<0.001$ \\
\hline ILF/IFO/optic radiations & Left & -22 & -78 & 0 & $416(29)$ & $440(21)$ & $<0.001$ \\
\hline Anterior thalamic radiation & Right & 20 & 6 & 10 & 435 (33) & $456(24)$ & 0.002 \\
\hline Corpus callosum & Right & 16 & 28 & 12 & $392(35)$ & $416(31)$ & $<0.001$ \\
\hline SLF & Right & 27 & 19 & 28 & 338 (29) & $362(23)$ & $<0.001$ \\
\hline SLF & Left & -31 & -26 & 32 & $368(30)$ & 389 (23) & $<0.001$ \\
\hline Corona radiata & Right & 14 & 9 & 44 & $423(43)$ & 455 (34) & 0.002 \\
\hline
\end{tabular}

compared with 0.323 in controls; $P=0.001$, Mann-Whitney test); the mean segmented white matter volume did not differ between the groups.

\section{Effects of illness duration and antipsychotic medication}

In our secondary analyses there was no significant correlation between the mean fractional anisotropy extracted from any of the clusters and duration of illness in the patient group. There was no significant difference between the mean fractional anisotropy extracted from any of the clusters between the briefly medicated and chronically medicated groups. Mean white matter fractional anisotropy over the whole brain likewise showed no correlation with illness duration (Fig. 2) and no medication-status group difference.

\section{Discussion}

Our principal analysis clearly indicated that fractional anisotropy was reduced in several regions of white matter in people with

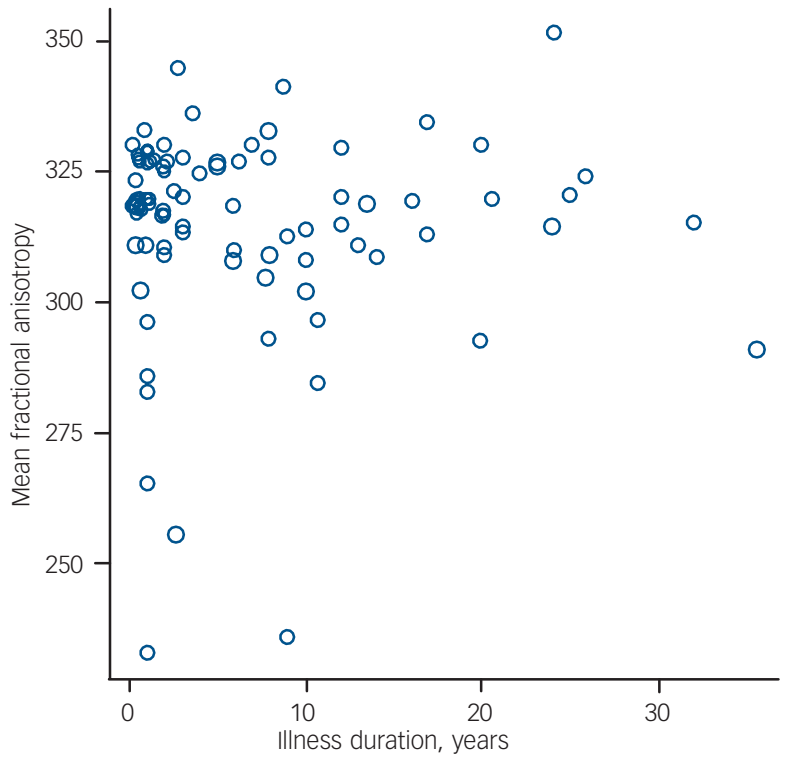

Fig. 2 Scatter plot of illness duration against mean segmented white-matter fractional anisotropy in patients with schizophrenia. schizophrenia, with no area of increased fractional anisotropy. This direction of differences is consistent with virtually all previous studies. ${ }^{6}$ The reductions were evident in areas of the white matter in both hemispheres, involving most of the commissural and association pathways in the brain. This would provide a correlate of, and potential pathological substrate for, the extremely diverse deficits in schizophrenia - phenomenologically, cognitively ${ }^{17}$ and structurally. ${ }^{18}$

Our results included most of the regions that have been reported in previous DTI studies such as the superior longitudinal fasciculus, ${ }^{19-23}$ the inferior longitudinal fasciculus, ${ }^{21}$ the corpus callosum $^{21,23-26}$ and the fornix. ${ }^{11}$ Our results can be argued to reconcile these previous discrepant findings. A systematic review of 19 anisotropy-based studies of schizophrenia by our group ${ }^{6}$ found anisotropy reductions reported over the whole brain, ${ }^{27}$ in regions of the corpus callosum, ${ }^{24,25,28}$ the cingulum, ${ }^{24,29-31}$ the superior longitudinal fasciculus, ${ }^{20,21}$ the uncinate fasciculus, ${ }^{21}$ frontal white matter, ${ }^{32}$ occipital white matter, ${ }^{32}$ the cerebellum ${ }^{33}$ and the hippocampus. ${ }^{34}$ There was a marked inconsistency in findings, however, which we speculated was due to small differences examined in small, heterogeneous samples with variable regions of interest: regions identified in one study would not be examined in most studies, or in underpowered ones, leading to false negatives. In the study reported here all of these regions were implicated, with the exception of the uncinate, even at a highly conservative threshold. This suggests that our study was able to detect almost all of the abnormalities that had been individually identified in earlier studies. This may reflect the greater statistical power afforded by relatively large, well-matched samples, and optimised acquisition, as well as the use of a voxel-based approach which permitted analysis of the entire white matter rather than selected regions within it. A recent study of a large sample with different methodology - using regions of interest on multiple tracts - found similarly widespread reductions, again with a question mark over the uncinate. ${ }^{35}$

Our secondary analysis revealed that, within the patient group, none of the reductions in fractional anisotropy was significantly related to the duration of psychotic illness. Recent cross-sectional and longitudinal MRI studies have provided evidence that at least some of the global and regional abnormalities of grey matter volume in schizophrenia progress over the course of the disorder. $^{3,36-43}$ Although some studies have found white matter volume deficits in schizophrenia, ${ }^{44}$ these are generally less pronounced than in grey matter, ${ }^{18}$ although they are also less well studied, ${ }^{4}$ and there is little evidence that these are progressive. ${ }^{41}$ Our finding that fractional anisotropy did not correlate with 
illness duration offers a microstructural counterpart to this. This has been reported previously, ${ }^{11,25,29,45}$ but these studies involved much smaller samples. Because our sample was relatively large and included patients with a range of illness durations, it is less likely that the absence of a correlation simply reflects limited statistical power. However, a recent, somewhat larger study by Mori et al found a small negative (uncorrected) correlation of fractional anisotropy with duration. ${ }^{46}$ One possible explanation for this discrepancy may lie with the different ages of the samples. Age is typically strongly correlated with duration of illness (there was a correlation coefficient of 0.685 in our study), and is itself related to fractional anisotropy in both patients and controls, ${ }^{47}$ although the relationship is perhaps non-linear. In early life, myelination - one of the determinants of fractional anisotropy tends to increase before declining in early-middle life. ${ }^{48}$ The sample investigated by Mori et al was relatively old (mean age 39 years) compared with our patients' mean age of 30 years, so the age-related decline might have been more pronounced. This may also partly explain the findings of Friedman et $a l^{47}$ who found widespread differences between patients with chronic schizophrenia and controls, but less extensive differences in a first-episode group (although they did not formally compare their chronic disorder and first-onset groups): again, their chronic disorder group was older than ours, with an age range extending beyond 80 years.

The relationship of illness duration and fractional anisotropy is also potentially confounded by the effect of antipsychotic medication, as in general the longer the duration of the disorder the longer the period of antipsychotic treatment. Antipsychotic medication has been linked to changes in grey matter and white matter volume in monkeys, ${ }^{49}$ and to grey matter reductions in humans. ${ }^{50}$ There is also evidence that, in humans at least, typical and atypical antipsychotic drugs affect brain volume differentially. ${ }^{51}$ There have been fewer studies of the effects of medication on white matter volume in schizophrenia and the results have been inconsistent. ${ }^{50,51}$ In our study there was no significant difference in regional or global white matter fractional anisotropy when chronically treated patients were compared with a matched group of patients who had received little or no medication, suggesting that antipsychotic medication did not affect white matter microstructure as measured by fractional anisotropy. This has also been reported previously, again in much smaller studies, ${ }^{25,29,45}$ although not all studies agreed, ${ }^{11}$ and one recent study reported fractional anisotropy decreases in a larger group who had received no medication at all. ${ }^{52}$ Our groups were matched for age, so age should not have confounded the analysis. Our findings are largely limited to atypical antipsychotic medication, however, since only one member of our combined sample was taking a typical antipsychotic drug. Our chronically medicated group had been treated for a median of 3 years, by which time most macrostructural effects had been demonstrated in other studies. ${ }^{51}$ However, most volume effects have been shown in the initial months, ${ }^{51}$ so that some medication effects cannot be entirely ruled out in our 'unmedicated' group, even though they had taken antipsychotics for a median of only 3 days.

Whole brain white matter fractional anisotropy was lower in our study, and this was not simply the effect of a few clusters of greatly reduced fractional anisotropy: $85 \%$ of white matter voxels had a mean fractional anisotropy lower in patients than in controls when the comparison was unthresholded (Fig. 3). However, our analysis suggests that if the fractional anisotropy reduction affected most white matter then, at the very least, there were areas that were more severely affected. Areas such as the uncinate $^{46}$ or the posterior cingulum, ${ }^{53}$ which other studies identified but our study did not, were on the edge of our clusters, and would have been included were our threshold less stringent.

\section{Limitations}

Although our large sample provided sufficient power to examine the effects of illness duration and medication on fractional anisotropy in a cross-sectional design, duration of illness and treatment are usually highly correlated. Differentiating their effects from each other, and from age, might be facilitated by prospective DTI studies, particularly in groups in whom it is possible to carefully monitor exposure to antipsychotic medication such as first-episode or high-risk samples.

One particular difficulty with the interpretation of our results lies in localising the differences. This is a difficulty in common with all voxel-based analysis methods. Region-of-interest and tractographic methods, although they face other challenges, ${ }^{7}$ at least have an a priori location within a specific tract or tracts. One area of difference from our results, for example, lies in the (a)

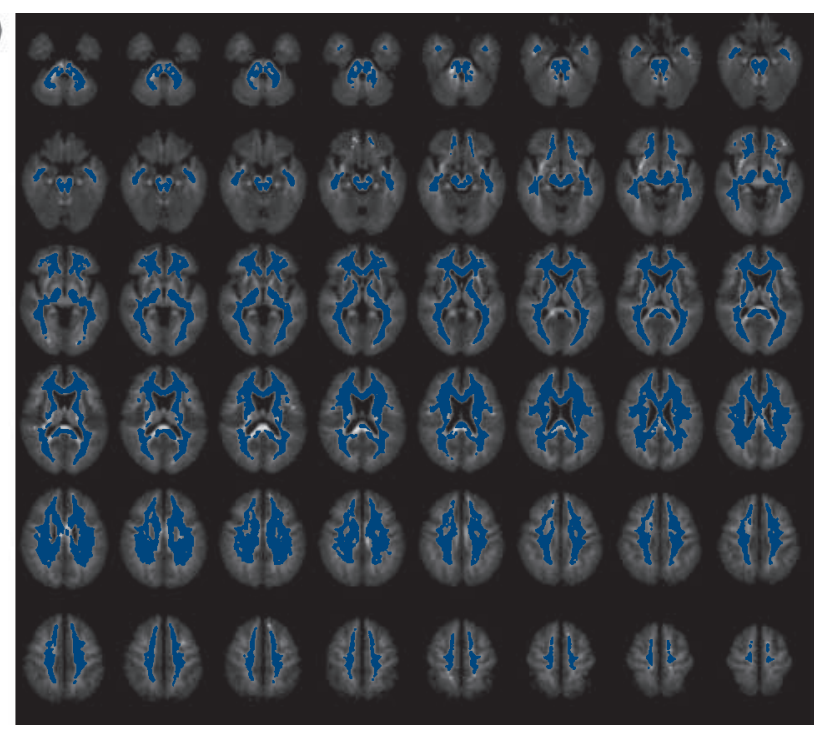

(b)

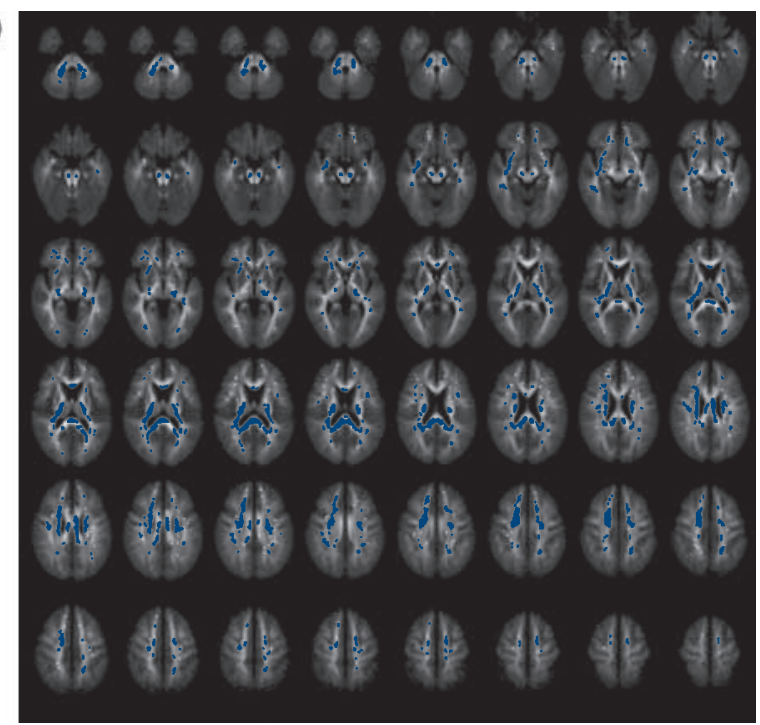

Fig. 3 White matter voxels where mean fractional anisotropy is lower (a) and higher (b) in patients than in controls (unthresholded comparison). 
right frontal white matter (Fig. 1), but it is unclear how best to describe this location, for it does not precisely correspond to any particular white matter tract: it lies largely in the genu of the corpus callosum, but morphologically is more like the anterior cingulum or the superior fronto-occipital fasciculus. This area has been highlighted in other studies, ${ }^{28,32}$ and has usually been described as 'the region of the cingulum'. Although this facilitates a functional interpretation, it would (in our case at least) ignore the bulk of the cluster. This reflects two issues facing DTI. The first is that white matter localisation lacks the precision and consensus that grey matter analysis has been used to for some time, although there are some notable attempts to rectify this, for example by using post-mortem samples. ${ }^{54}$ The second is that a cluster- or voxel-based analysis is designed to identify clusters or voxels of difference rather than tracts. Clusters will not necessarily lie within single tracts, even where they can be readily distinguished from their neighbours, and this makes the description and interpretation of the results more difficult. Although it is tempting to localise a cluster to a single tract for these reasons, this assumes that any true difference corresponds to the morphology of a tract, and that the cluster is only a partial capture of that true difference. The reality may be very different, however, and is likely to vary with the causes of any white matter changes. A tract-like difference might be predicted where white matter disconnectivity has developed in response to altered function. However, environmental insults might follow the pattern of arterial supply, for example, or of infective foci, leading to clusters of difference (as in progressive multifocal leukoencephalopathy). ${ }^{55}$ Moreover, genetic abnormalities, in myelination for example, might lead to differences that affected all white matter, to a greater or lesser degree (as in adrenoleukodystrophy). ${ }^{56}$ Our results, which are supportive of cluster-level or whole-brain effects, must be interpreted in the context of the analysis method.

In conclusion, our findings indicate that there are widespread reductions in fractional anisotropy in schizophrenia, independent of illness duration and the effects of antipsychotic medication. There are various determinants of reduced fractional anisotropy, the most important of which are disordered neuronal architecture and myelination, ${ }^{57,58}$ and there is evidence for both of these in schizophrenia. ${ }^{59-61}$ Since either of these would lead to altered white matter function, our finding of reduced fractional anisotropy provides support for disconnectivity models of schizophrenia. Further, since the differences were so widespread, they would support disconnectivity between frontal, temporal, parietal, occipital and cerebellar regions.

\footnotetext{
Richard Kanaan, MRCPsych, Gareth Barker, PhD, Michael Brammer, PhD, Vincent Giampietro, PhD, Sukhwinder Shergill, MRCPsych, PhD, James Woolley, MRCPsych, Marco Picchioni, MRCPSych, Timothea Toulopoulou, PhD, Woolley, MRCPSych, Marco Picchioni, MRCPsych, Timothea Toulopoulou, PhD,
Philip McGuire, FRCPsych, PhD, King's College London, Institute of Psychiatry, London, UK

Correspondence: Dr Richard Kanaan, Institute of Psychiatry, Department of Psychological Medicine, PO 62, Denmark Hill, London SE5 9RJ, UK. Email: r.kanaan@iop.kcl.ac.uk

First received 9 May 2008, final revision 21 Oct 2008, accepted 23 Oct 2008
}

\section{Acknowledgements}

R.K., M.P. and S.S. were funded by The Wellcome Trust. P.M. has received honoraria for lectures and consultancy fees from Lilly, Astrazeneca and Janssen Cilag. S.S. has received honoraria or support to attend conferences from Janssen, Lilly, Novartis and SanofiSynthelabo, and unrestricted project grant support from Novartis and AstraZeneca.

\section{References}

1 Stephan KE, Baldeweg T, Friston KJ. Synaptic plasticity and dysconnection in schizophrenia. Biol Psychiatry 2006; 59: 929-39.
2 Friston KJ. Dysfunctional connectivity in schizophrenia. World Psychiatry 2002; 1: 66-71.

3 Velakoulis D, Wood SJ, Wong MT, McGorry PD, Yung A, Phillips L, et al. Hippocampal and amygdala volumes according to psychosis stage and diagnosis: a magnetic resonance imaging study of chronic schizophrenia, first-episode psychosis, and ultra-high-risk individuals. Arch Gen Psychiatry 2006; 63: 139-49.

4 Walterfang M, Wood SJ, Velakoulis D, Pantelis C. Neuropathological, neurogenetic and neuroimaging evidence for white matter pathology in schizophrenia. Neurosci Biobehav Rev 2006; 30: 918-48.

5 Basser PJ, Mattiello J, LeBihan D. Estimation of the effective self-diffusion tensor from the NMR spin echo. J Magn Reson B 1994; 103: 247-54.

6 Kanaan RA, Kim JS, Kaufmann WE, Pearlson GD, Barker GJ, McGuire PK. Diffusion tensor imaging in schizophrenia. Biol Psychiatry 2005; 58: 921-9.

7 Kanaan RA, Shergill SS, Barker GJ, Catani M, Ng VW, Howard R, et al. Tractspecific anisotropy measurements in diffusion tensor imaging. Psychiatry Res 2006; 146: 73-82.

8 Jones DK, Williams SC, Gasston D, Horsfield MA, Simmons A, Howard R. Isotropic resolution diffusion tensor imaging with whole brain acquisition in a clinically acceptable time. Hum Brain Mapp 2002; 15: 216-30.

9 American Psychiatric Association. Diagnostic and Statistical Manual of Mental Disorders (4th edn) (DSM-IV). APA, 1994.

10 Russell AJ, Munro J, Jones PB, Hayward P, Hemsley DR, Murray RM. The National Adult Reading Test as a measure of premorbid IQ in schizophrenia. Br J Clin Psychol 2000; 39: 297-305.

11 Kuroki N, Kubicki M, Nestor PG, Salisbury DF, Park HJ, Levitt JJ, et al. Fornix integrity and hippocampal volume in male schizophrenic patients. Biol Psychiatry 2006; 60: 22-31.

12 Jones DK, Symms M, Cercignani M, Howard R. The effect of filter size on the outcome of VBM analyses of DT-MRI data. Neuroimage 2005; 26: 546-54.

13 Rabe-Hesketh S, Bullmore ET, Brammer MJ. The analysis of functional magnetic resonance images. Stat Methods Med Res 1997; 6: 215-37.

14 Poline JB, Worsley KJ, Evans AC, Friston KJ. Combining spatial extent and peak intensity to test for activations in functional imaging. Neuroimage 1997; 5: 83-96.

15 Mori S, Wakana S, Van Zijl PCM. MRI Atlas of Human White Matter. Elsevier, 2005.

16 Crosby EC, Humphrey T, Lauer EW. Correlative Anatomy of the Nervous System. Macmillan, 1962.

17 Reichenberg A, Harvey PD. Neuropsychological impairments in schizophrenia: integration of performance-based and brain imaging findings. Psychol Bull 2007; 133: 833-58.

18 Davatzikos C, Shen D, Gur RC, Wu X, Liu D, Fan Y, et al. Whole-brain morphometric study of schizophrenia revealing a spatially complex set of focal abnormalities. Arch Gen Psychiatry 2005; 62: 1218-27.

19 Buchsbaum MS, Friedman J, Buchsbaum BR, Chu KW, Hazlett EA, Newmark $\mathrm{R}$, et al. Diffusion tensor imaging in schizophrenia. Biol Psychiatry 2006; 60: 1181-7.

20 Burns J, Job D, Bastin ME, Whalley H, Macgillivray T, Johnstone EC, et al. Structural disconnectivity in schizophrenia: a diffusion tensor magnetic resonance imaging study. Br J Psychiatry 2003; 182: 439-43.

21 Hubl D, Koenig T, Strik W, Federspiel A, Kreis R, Boesch C, et al. Pathways that make voices: white matter changes in auditory hallucinations. Arch Gen Psychiatry 2004; 61: 658-68.

22 Jones DK, Catani M, Pierpaoli C, Reeves SJ, Shergill SS, O'Sullivan M, et al. Age effects on diffusion tensor magnetic resonance imaging tractography measures of frontal cortex connections in schizophrenia. Hum Brain Mapp 2006; 27: 230-8.

23 Shergill SS, Kanaan RA, Chitnis XA, O'Daly O, Jones DK, Frangou S, et al A diffusion tensor imaging study of fasciculi in schizophrenia. Am J Psychiatry 2007; 164: 467-73.

24 Agartz I, Andersson JL, Skare S. Abnormal brain white matter in schizophrenia: a diffusion tensor imaging study. Neuroreport 2001; 12 2251-4.

25 Foong J, Maier M, Clark CA, Barker GJ, Miller DH, Ron MA. Neuropathological abnormalities of the corpus callosum in schizophrenia: a diffusion tensor imaging study. J Neurol Neurosurg Psychiatry 2000; 68: 242-4.

26 Price G, Cercignani M, Parker GJ, Altmann DR, Barnes TR, Barker GJ, et al. Abnormal brain connectivity in first-episode psychosis: a diffusion MRI tractography study of the corpus callosum. Neuroimage 2007; 35: 458-66.

27 Lim KO, Hedehus M, Moseley M, de Crespigny A, Sullivan EV, Pfefferbaum A. Compromised white matter tract integrity in schizophrenia inferred from diffusion tensor imaging. Arch Gen Psychiatry 1999; 56: 367-74. 
28 Ardekani BA, Nierenberg J, Hoptman MJ, Javitt DC, Lim KO. MRI study of white matter diffusion anisotropy in schizophrenia. Neuroreport 2003; 14 2025-9.

29 Kubicki M, Westin CF, Nestor PG, Wible CG, Frumin M, Maier SE, et al. Cingulate fasciculus integrity disruption in schizophrenia: a magnetic resonance diffusion tensor imaging study. Biol Psychiatry 2003; 54: 1171-80.

30 sun $Z$, Wang F, Cui L, Breeze J, Du X, Wang X, et al. Abnormal anterior cingulum in patients with schizophrenia: a diffusion tensor imaging study. Neuroreport 2003; 14: 1833-6.

31 Wang $F$, Sun Z, Cui L, Du X, Wang X, Zhang $H$, et al. Anterior cingulum abnormalities in male patients with schizophrenia determined through diffusion tensor imaging. Am J Psychiatry 2004; 161: 573-5.

32 Kumra S, Ashtari M, McMeniman M, Vogel J, Augustin R, Becker DE, et al. Reduced frontal white matter integrity in early-onset schizophrenia: a preliminary study. Biol Psychiatry 2004; 55: 1138-45.

33 Wang $F$, Sun $Z$, Du $X$, Wang $X$, Cong $Z$, Zhang $H$, et al. A diffusion tensor imaging study of middle and superior cerebellar peduncle in male patients with schizophrenia. Neurosci Lett 2003; 348: 135-8.

34 Kalus P, Buri C, Slotboom J, Gralla J, Remonda L, Dierks T, et al. Volumetry and diffusion tensor imaging of hippocampal subregions in schizophrenia. Neuroreport 2004; 15: 867-71.

35 Mitelman SA, Torosjan Y, Newmark RE, Schneiderman JS, Chu KW, Brickman AM, et al. Internal capsule, corpus callosum and long associative fibers in good and poor outcome schizophrenia: a diffusion tensor imaging survey. Schizophr Res 2007; 92: 211-24.

36 Kasai K, Shenton ME, Salisbury DF, Hirayasu Y, Onitsuka T, Spencer MH, et al. Progressive decrease of left Heschl gyrus and planum temporale gray matter volume in first-episode schizophrenia: a longitudinal magnetic resonance imaging study. Arch Gen Psychiatry 2003; 60: 766-75.

37 Kasai $K$, Shenton ME, Salisbury DF, Onitsuka T, Toner SK, Yurgelun-Todd D, et al. Differences and similarities in insular and temporal pole MRI gray matter volume abnormalities in first-episode schizophrenia and affective psychosis Arch Gen Psychiatry 2003; 60: 1069-77.

38 Cahn W, Hulshoff Pol HE, Lems EB, van Haren NE, Schnack HG, van der Linden JA, et al. Brain volume changes in first-episode schizophrenia: a 1year follow-up study. Arch Gen Psychiatry 2002; 59: 1002-10.

39 Cahn W, van Haren NEM, Hulshoff Pol HE, Schnack HG, Caspers E, Laponde DAJ, et al. Brain volume changes in the first year of illness and 5-year outcome of schizophrenia. Br J Psychiatry 2006; 189: 381-2.

40 Ho BC, Andreasen NC, Nopoulos P, Arndt S, Magnotta V, Flaum M. Progressive structural brain abnormalities and their relationship to clinical outcome: a longitudinal magnetic resonance imaging study early in schizophrenia. Arch Gen Psychiatry 2003; 60: 585-94.

41 Van Haren NE, Hulshoff Pol HE, Schnack HG, Cahn W, Mandl RC, Collins DL, et al. Focal gray matter changes in schizophrenia across the course of the illness: a 5-year follow-up study. Neuropsychopharmacology 2007; 32: 2057-66.

42 Velakoulis D, Wood SJ, Smith DJ, Soulsby B, Brewer W, Leeton L, et al. Increased duration of illness is associated with reduced volume in right medial temporal/anterior cingulate grey matter in patients with chronic schizophrenia. Schizophr Res 2002; 57: 43-9.

43 Wood SJ, Velakoulis D, Smith DJ, Bond D, Stuart GW, McGorry PD, et al. A longitudinal study of hippocampal volume in first episode psychosis and chronic schizophrenia. Schizophr Res 2001; 52: 37-46.

44 Wright IC, Rabe-Hesketh S, Woodruff PW, David AS, Murray RM, Bullmore ET. Meta-analysis of regional brain volumes in schizophrenia. Am J Psychiatry 2000; 157: 16-25.
45 Kubicki M, Westin CF, Maier SE, Frumin M, Nestor PG, Salisbury DF, et al. Uncinate fasciculus findings in schizophrenia: a magnetic resonance diffusion tensor imaging study. Am J Psychiatry 2002; 159: 813-20.

46 Mori $T$, Ohnishi $T$, Hashimoto $R$, Nemoto $K$, Moriguchi $Y$, Noguchi $H$, et al. Progressive changes of white matter integrity in schizophrenia revealed by diffusion tensor imaging. Psychiatry Res 2007; 154: 133-45.

47 Friedman Jl, Tang C, Carpenter D, Buchsbaum M, Schmeidler J, Flanagan L, et al. Diffusion tensor imaging findings in first-episode and chronic schizophrenia patients. Am J Psychiatry 2008; 165: 1024-32.

48 Bartzokis G, Beckson M, Lu PH, Nuechterlein KH, Edwards N, Mintz J. Agerelated changes in frontal and temporal lobe volumes in men: a magnetic resonance imaging study. Arch Gen Psychiatry 2001; 58: 461-5.

49 Dorph-Petersen KA, Pierri JN, Perel JM, Sun Z, Sampson AR, Lewis DA. The influence of chronic exposure to antipsychotic medications on brain size before and after tissue fixation: a comparison of haloperidol and olanzapine in macaque monkeys. Neuropsychopharmacology 2005; 30: 1649-61.

50 Molina V, Reig S, Sanz J, Palomo T, Benito C, Sanchez J, et al. Increase in gray matter and decrease in white matter volumes in the cortex during treatment with atypical neuroleptics in schizophrenia. Schizophr Res 2005; 80: 61-71.

51 Lieberman JA, Tollefson GD, Charles C, Zipursky R, Sharma T, Kahn RS, et al. Antipsychotic drug effects on brain morphology in first-episode psychosis. Arch Gen Psychiatry 2005; 62: 361-70.

52 Cheung V, Cheung C, McAlonan GM, Deng Y, Wong JG, Yip L, et al. A diffusion tensor imaging study of structural dysconnectivity in never-medicated, firstepisode schizophrenia. Psychol Med 2008; 38: 877-85.

53 Fujiwara $\mathrm{H}$, Namiki C, Hirao K, Miyata J, Shimizu M, Fukuyama $\mathrm{H}$, et al. Anterior and posterior cingulum abnormalities and their association with psychopathology in schizophrenia: a diffusion tensor imaging study. Schizophr Res 2007; 95: 215-22.

54 Burgel U, Amunts K, Hoemke L, Mohlberg H, Gilsbach JM, Zilles K. White matter fiber tracts of the human brain: three-dimensional mapping at microscopic resolution, topography and intersubject variability. Neuroimage 2006; 29: 1092-105

55 Kuker W, Mader I, Nagele T, Uhl M, Adolph C, Klose U, et al. Progressive multifocal leukoencephalopathy: value of diffusion-weighted and contrastenhanced magnetic resonance imaging for diagnosis and treatment control. Eur J Neurol 2006; 13: 819-26.

56 Schneider JF, II'yasov KA, Boltshauser E, Hennig J, Martin E. Diffusion tensor imaging in cases of adrenoleukodystrophy: preliminary experience as a marker for early demyelination? AJNR 2003; 24: 819-24.

57 Pierpaoli C, Basser PJ. Toward a quantitative assessment of diffusion anisotropy. Magn Reson Med 1996; 36: 893-906.

58 Beaulieu $C$. The basis of anisotropic water diffusion in the nervous system a technical review. NMR Biomed 2002; 15: 435-55.

59 Flynn SW, Lang DJ, Mackay AL, Goghari V, Vavasour IM, Whittall KP, et al. Abnormalities of myelination in schizophrenia detected in vivo with MRI, and post-mortem with analysis of oligodendrocyte proteins. Mol Psychiatry 2003; 8: 811-20.

60 Akbarian S, Kim JJ, Potkin SG, Hetrick WP, Bunney WE, Jones EG. Maldistribution of interstitial neurons in prefrontal white matter of the brains of schizophrenic patients. Arch Gen Psychiatry 1996; 53: 425-36.

61 Kubicki M, Park H, Westin CF, Nestor PG, Mulkern RV, Maier SE, et al. DTI and MTR abnormalities in schizophrenia: analysis of white matter integrity. Neuroimage 2005; 26: 1109-18. 